\title{
External triggering of substorms identified using modern optical versus geosynchronous particle data
}

\author{
B. Gallardo-Lacourt ${ }^{1}$, Y. Nishimura ${ }^{1}$, L. R. Lyons ${ }^{1}$, and E. Donovan ${ }^{2}$ \\ ${ }^{1}$ Department of Atmospheric and Oceanic Sciences, University of California, Los Angeles, USA \\ ${ }^{2}$ Department of Physics and Astronomy, University of Calgary, Calgary, Alberta, Canada \\ Correspondence to: B. Gallardo-Lacourt (bgallardo@atmos.ucla.edu)
}

Received: 20 September 2011 - Revised: 19 December 2011 - Accepted: 7 February 2012 - Published: 4 April 2012

\begin{abstract}
Previous works on substorm triggering have shown that more than $50 \%$ of the substorms are triggered by a northward turning of the IMF $B_{\mathrm{Z}}$; However, recent studies have found a much lower percentage. We have examined triggering using three different onset lists: The THEMIS All Sky Image (ASI) list, substorm onsets from IMAGE-FUV, and events with large geosynchronous injections. We analyzed these onset lists with three different triggering criteria: (1) a criteria based on Lyons et al. criteria; (2) a relaxation of the Lyons et al. criteria based on the visual criteria proposed by Hsu et al.; and (3) a further relaxation of the Lyons et al. criteria, requiring the same conditions proposed in the visual criteria by Hsu et al. but without the growth phase southward IMF requirements. Appling the Lyons et al. criteria we find that only $17 \%$ and $22 \%$ of the substorms are triggered in the THEMIS ASIs and IMAGE-FUV onset lists respectively, consistent with the recent studies. However, the percentage reached nearly $50 \%$ when we applied relaxed criteria, suggesting that it is possible that the Lyons et al. criteria are too strict to identify all IMF triggered events. The triggering percentage for the events with large injections reached up to $60 \%$ applying the relaxed criteria, a result suggesting the possibility that triggers are more easily identified, or that triggering is more common for larger than for smaller substorm events. We have also found evidence that larger substorms may be more likely to be non-triggered under mostly southward IMF conditions than for other IMF conditions.
\end{abstract}

Keywords. Magnetospheric physics (Auroral phenomena; Magnetotail; Storms and substorms)

\section{Introduction}

Substorms are dramatic disturbances of the global magnetosphere-ionosphere system during which solar wind energy stored in the magnetotail is released (Rostoker et al., 1980). The onset of substorms is identifiable by auroral brightening along an arc near the equatorward boundary of the auroral oval and likely maps along magnetic field lines toward the near-Earth portion of the plasma sheet (Samson et al., 1992; Nishimura et al., 2010). An important question for understanding substorms is whether substorm onset is the result of a change in the external conditions or the result of an instability that is purely internal to the magnetosphere. The primary external factor that has been considered as a cause of onset is a turning towards the north in the direction of the Interplanetary Magnetic Field (IMF), since the IMF direction is the dominant modulator of the strength of solar wind coupling to the magnetosphere. However, there has been a long debate as to the extent to which substorms are externally triggered by IMF changes. Even after many reports of an association between northward turning of the IMF and substorm onset (Foster et al., 1971; Caan et al., 1975, 1977; Rostoker, 1983; McPherron et al., 1986), cases without northward turning have also been presented (e.g., Henderson et al., 1996) and thus the external triggering of substorms is still a very controversial issue. Lyons et al. (1997) proposed quantitative criteria of the IMF $B_{\mathrm{Z}}$ changes that are effective for substorm triggering (see Sect. 2 for criteria details). Using the criteria, they found a high occurrence probability of triggering for a limited number of events ( 14 of 20 events, $70 \%$ ). 
Hsu and McPherron (2003) examined the triggering hypothesis using an onset list based on the AL index and Pi 2 pulsations. They used two different approaches, the Lyons et al. criteria and a visual inspection, which is essentially a relaxed version of the Lyons et al. criteria (see Sect. 2 for details), and determined the occurrence of external substorm triggering. They obtained a remarkably high $(60 \%)$ triggering percentage using the visual inspection, while it was much lower $(33 \%)$ for the Lyons et al. criteria. This result suggests that the Lyons et al. criteria may be too strict for identifying triggering, or that triggering occurs less frequently than obtained by Hsu et al.

Statistical analysis related to events occurring randomly in space and time (e.g., Cox, 1955; Hsu and McPherron, 2002) were used by Morley and Freeman (2007) to analyze the association between IMF triggering and substorm onsets, and they did not find significant evidence for triggering, in contrast to the finding by Lyons et al. A recent study by Newell and Liou (2011) also showed a low occurrence probability (24\%) of triggering using the same triggering criteria used by Lyons et al. In their study, the triggering percentage for the onset list created by Frey et al. (2004) was compared to triggering percentages for completely random epochs and quasi-random epochs. Similar triggering percentages were obtained for all three types, suggesting that northward turnings may not be a significant trigger for substorm onset.

As can be seen in the studies mentioned above, the issue of substorm external triggering still remains controversial. Although the Lyons et al. criteria have been shown to give low triggering occurrences (Hsu and McPherron, 2003; Morley and Freeman, 2007; Newell and Liou, 2011), the attempt of the visual inspection by Hsu et al. suggests that more triggered events could be found by adjusting the triggering criteria. This would be plausible because the Lyons et al. criteria are based on a limited number of events and could indicate that triggering by northward turnings might indeed be important. In the present paper, we examine the triggering hypothesis by applying three different triggering criteria to three different lists of substorms. We choose three different criteria because different criteria have been used in the above earlier studies, and it is not known which is the most realistic. Thus a result that holds for all of these criteria would indicate that the result is not dependent on the specifics of the criteria and is therefore more likely to be correct than a result that is found for only one set of criteria. The different onset lists identify substorms with different minimum strengths, the smallest being by high-resolution, ground-based imaging and the largest being the traditional identification by large dispersionless geosynchronous particle injection events. We show that the triggering percentage is significantly higher when applying relaxed criteria and including IMF $B_{\mathrm{y}}$ triggering, suggesting that the external triggering could be common even while the Lyons et al. criteria give a low percentage. Furthermore, the large injection events are found to give higher triggering occurrence than the lists which include weaker substorms, indicating that triggering may be more likely for larger events and that the corresponding IMF orientation changes may be larger and thus more easily identified.

\section{Methodology}

This study uses three different lists of substorm onsets. The THEMIS All Sky Image (ASI) list (Nishimura et al., 2010) includes 190 isolated auroral onset events obtained from November 2007 to April 2008. The THEMIS ASIs have the capability to detect substorm auroral onsets in high spatial and temporal resolution as well as high sensitivity. This advantage allows us to obtain onset timing unambiguously and identify weak substorms that were maybe missed in the AL index and space-based auroral imaging. The ASIs can also distinguish other types of auroral disturbances (e.g., poleward boundary intensifications and auroral streamers) that lead to ground magnetic field perturbations similar to those from substorms. The present analysis focuses on isolated onsets, which occur more than $30 \mathrm{~min}$ after preceding onsets.

The second list of onsets used in this analysis is composed of substorm onsets observed by the IMAGE-FUV from May 2000 to December 2002 (Frey et al., 2004). The FUV imager on board the IMAGE spacecraft provides global auroral imaging in the far ultraviolet. This list has a large number (2437) of events, which help to increase the statistical significance. This list was also used for the IMF triggering study by Newell and Liou (2011).

The third list of onsets corresponds to 64 events of large electron injection from Los Alamos National Laboratory (LANL) geosynchronous energetic particle data obtained from January 2000 to April 2001. Here, electron injections were analyzed using the Synchronous Orbit Particle Analyzer (SOPA) instrument which measures electrons from $50 \mathrm{keV}$ to $26 \mathrm{MeV}$ in 16 energy channels. Although the injection events do not have supportive auroral observations, rapid increases in energetic particle fluxes without energy dispersion are known as a substorm-related phenomenon (Reeves, 1996). As a list of large substorms, we selected large, dispersionless injection events, where electron fluxes at one of the spacecraft for any two energy channels were enhanced by more than one order of magnitude. Dispersionless injections seen at more than one spacecraft were not included because they can result from the magnetospheric compressions from solar wind dynamic pressure enhancements. Solar wind parameters were obtained from the OMNI database with $1 \mathrm{~min}$ resolution.

We use three different set of criteria for external triggering of substorms. The first is based on the Lyons et al. (1997) criteria, and three major categories are established: $B_{\mathrm{z}}$-triggered, $B_{\mathrm{y}}$-triggered, and non-triggered. $B_{\mathrm{z}^{-}}$ triggered events represent clear northward turning of the IMF $B_{\mathrm{Z}}$ by more than $2 \mathrm{nT}$ with respect to the average value for 


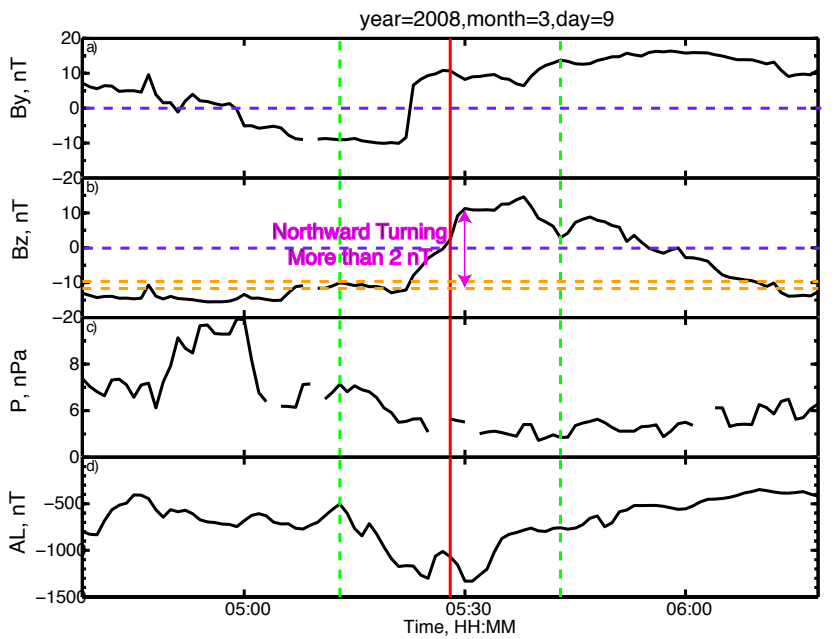

Fig. 1. Example of a $B_{\mathrm{Z}}$-triggered event applying the criteria based on Lyons et al. The first panel (a) represents $B_{\mathrm{y}}$, (b) shows $B_{\mathrm{z}}$, (c) indicates dynamic pressure and (d) AL index. The red line indicates the onset time. The two vertical green lines indicate $\pm 15 \mathrm{~min}$ around the onset time. The horizontal orange lines indicate a $2 \mathrm{nT}$ change of the IMF $B_{\mathrm{Z}}$ starting at the initial point of the northward turning. The horizontal blue line indicates the location of zero for reference.

the 20 min preceding the northward turning, and this point should be located within $\pm 15 \mathrm{~min}$ of the onset time. $B_{\mathrm{Z}}$ should be negative with a standard deviation less than $2 \mathrm{nT}$ during the $20 \mathrm{~min}$ preceding the northward turning, and $B_{\mathrm{Z}}$ should remain more than $2 \mathrm{nT}$ above its average over the preceding $20 \mathrm{~min}$ for the $10 \mathrm{~min}$ following the northward turning. Figure 1 shows an example satisfying these criteria. The panels show (a) the IMF $B_{\mathrm{y}}$; (b) IMF $B_{\mathrm{z}}$; (c) dynamic pressure and (d) AL index. This event shows a significantly large northward turning starting at 05:22 UT and remaining elevated for more than $10 \mathrm{~min}$. If an event does not satisfying the $B_{\mathrm{Z}}$ criteria, it is considered if the magnitude of the IMF $B_{\mathrm{y}}$ decreases by at least $2 \mathrm{nT}$ from its average over the preceding $20 \mathrm{~min}$ and remains decreased by at least $2 \mathrm{nT}$ for $5 \mathrm{~min}$. Also, the standard deviation of $B_{\mathrm{y}}$ must be less than $2 \mathrm{nT}$ during the preceding $20 \mathrm{~min}$. Events with a sign change in $B_{\mathrm{y}}$ are also counted if $B_{\mathrm{y}}$ changes by more than $4 \mathrm{nT}$. Figure 2 provides an example of a $B_{\mathrm{y}}$ triggered event, the IMF $B_{\mathrm{Z}}$ showing a $\sim 2 \mathrm{nT}$ reduction starting at 06:05 UT while the IMF $B_{\mathrm{Z}}$ stayed essentially constant.

The rest of the events, which are classified as nontriggered, are divided into three subcategories: mostly south, mostly north, and fluctuating. The mostly south subdivision corresponds to events where the IMF $B_{\mathrm{Z}}$ remains negative for at least $75 \%$ of the 1 -h time period $( \pm 30 \mathrm{~min})$ around the substorm onset time. The events are called mostly north if the IMF $B_{\mathrm{Z}}$ is positive at least $75 \%$ of the 1 -h time period. Events that do not satisfy either of the above two criteria are called fluctuating. Figure 3 presents examples of

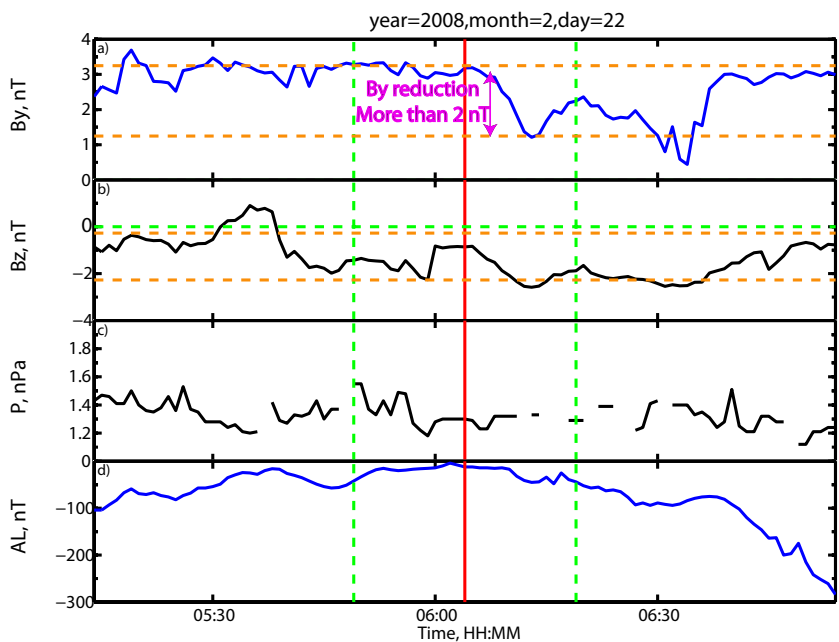

Fig. 2. Example of a $B_{y}$-triggered event in the same format as Fig. 1.

non-triggered events: (A) mostly south, (B) mostly north and (C) fluctuating.

We relax the Lyons et al. criteria for defining external triggering substorms in two ways. The first is based on the visual criteria proposed by Hsu and McPherron (2003). Their criteria follow the Lyons et al. criteria except that a northward turning by $2 \mathrm{nT}$ at one point is required to be detected within \pm 15 min of the substorm onset time and the IMF $B_{\mathrm{Z}}$ should remain elevated by any magnitude for $5 \mathrm{~min}$ after the northward turning. Figure 4 shows an example of a $B_{\mathrm{z}}$-triggered event applying the criteria based on the visual criteria established by Hsu and McPherron (2003). The northward turning occurring at 05:20 UT has a change of $\sim 3.5 \mathrm{nT}$ and remains elevated for $6 \mathrm{~min}$. This event is not counted in the Lyons et al. criteria because of the short duration of the elevated IMF but is included in the Hsu et al. definition.

As a further relaxation of the Lyons et al. criteria, we use the same requirements as for the Hsu et al. criteria above, but we do not require a steady southward IMF prior to the northward turning. However, a northward turning of the IMF $B_{\mathrm{Z}}$ by more than $2 \mathrm{nT}$ with respect to the average value for the 20 min preceding the northward turning is required. With this relaxation, a northward turning is counted as a trigger even if the preceding IMF is northward IMF. This allows for the possibility of a growth phase to develop under northward IMF, as can occur when there are large fluctuations in the IMF (Kim et al., 2009; Lyons et al., 2009), or when the IMF $B_{\mathrm{y}}$ is large. Figure 5 illustrates an example showing a northward turning starting at 02:21 UT with brief northward excursions prior to the northward turning. 
Table 1. Percentages of IMF triggering for the THEMIS events. The first row (A) represents the results applying the Lyons et al. criteria. The second row (B) provides the triggering percentage applying the Hsu et al. visual criteria. The third row (C) shows the results applying the same criteria used in row (B) but without requirements for the preceding IMF $B_{\mathrm{Z}}$. The values in the tables represent percentages.

\begin{tabular}{lccccc}
\hline & & & \multicolumn{2}{c}{ Non triggered } \\
\cline { 4 - 6 } & $B_{\mathrm{z}}$ & $B_{\mathrm{z}}+B_{\mathrm{y}}$ & Mostly South & Mostly North & Fluctuating \\
\hline (A) Lyons et al. & 17 & 22 & 32 & 21 & 25 \\
(B) Hsu et al. & 27 & 33 & 31 & 17 & 19 \\
(C) relaxed Hsu et al. & 36 & 41 & 29 & 16 & 14 \\
\hline
\end{tabular}

Table 2. Same as Table 1 but for the event list from IMAGE-FUV.

\begin{tabular}{lccccc}
\hline & & & \multicolumn{2}{c}{ Non triggered } \\
\cline { 4 - 6 } & $B_{\mathrm{Z}}$ & $B_{\mathrm{Z}}+B_{\mathrm{y}}$ & Mostly South & Mostly North & Fluctuating \\
\hline (A) Lyons et al. & 22 & 30 & 38 & 18 & 14 \\
(B) Hsu et al. & 32 & 40 & 33 & 17 & 13 \\
(C) relaxed Hsu et al. & 43 & 48 & 33 & 6 & 13 \\
\hline
\end{tabular}

Table 3. Same as Table 1 but for the large dispersionless injections.

\begin{tabular}{lcccccc}
\hline & & & \multicolumn{2}{c}{ Non triggered } \\
\cline { 4 - 6 } & $B_{\mathrm{Z}}$ & $B_{\mathrm{Z}}+B_{\mathrm{y}}$ & Mostly South & Mostly North & Fluctuating \\
\hline (A) Lyons et al. & 34 & 42 & 44 & 6 & 8 \\
(B) Hsu et al. & 40 & 54 & 33 & 6 & 7 \\
(C) relaxed Hsu et al. & 41 & 63 & 27 & 5 & 5 \\
\hline
\end{tabular}

\section{Results}

Tables 1-3 show the results for the statistical study for the onset lists applying the three different triggering criteria. The rows represent the triggering percentage applying the criteria of Lyons et al. (1997); Hsu and McPherron (2003) and Hsu et al. without requirement for the preceding southward IMF. The tables are divided into $B_{\mathrm{z}}$-triggered, $B_{\mathrm{z}}$ or $B_{\mathrm{y}}$-triggered and Non-triggered events. " $B_{\mathrm{z}}$ or $B_{\mathrm{y}}$-triggered" includes the $B_{\mathrm{z}}$-triggered events and $B_{\mathrm{y}}$-triggered events defined in the previous section, and thus indicates the total IMF triggered events. The non-triggered category is divided into three subcategories mostly south, mostly north and fluctuating according to the definitions established above. The values in the tables represent percentages.

Table 1 provides the results for the THEMIS ASI event list. The percentage of the $B_{\mathrm{Z}}$-triggered events is low (17\%) for the Lyons et al. criteria, while it is significantly higher $(27 \%)$ for Hsu et al. and $(36 \%)$ for Hsu et al. without the requirement of the preceding IMF $B_{\mathrm{z}}$. Similarly, the percentage of the $B_{\mathrm{z}}$ or $B_{\mathrm{y}}$-triggered events increases from 22 to $41 \%$ with relaxing the criteria. The total percentage of nontriggered events conversely decreases from $78 \%$ for Lyons et al. to $59 \%$ for $\mathrm{Hsu}$ et al. without requirement for preceding IMF $B_{\mathrm{Z}}$.

Table 2 provides the results for the list of onset from IMAGE-FUV. Consistently with the results in Table 1, the percentage of the triggered events significantly increases with relaxing the triggering criteria. The percentage of the $B_{\mathrm{Z}}$-triggered events is limited to $22 \%$ for Lyons et al. This is a similar percentage as and thus consistent with the finding by Newell and Liou (2011). In contrast, the percentage is significantly higher for the Hsu et al. (32\%) and relaxed Hsu et al. criteria $(43 \%)$. The triggering percentage increases more when including the $B_{\mathrm{y}}$-triggered events and reaches close to $50 \%$.

The results above indicate that almost half of the substorms may be triggered by IMF orientation changes by considering the relaxed criteria. While we can not ascertain whether or not the Lyons et al. criteria are too strict to identify many IMF triggering, this is a possibility to explain the changes in the triggering percentage. However, the other half of the events cannot be explained by the triggering hypothesis unless triggering occurs under conditions not envisioned in previous studies. 

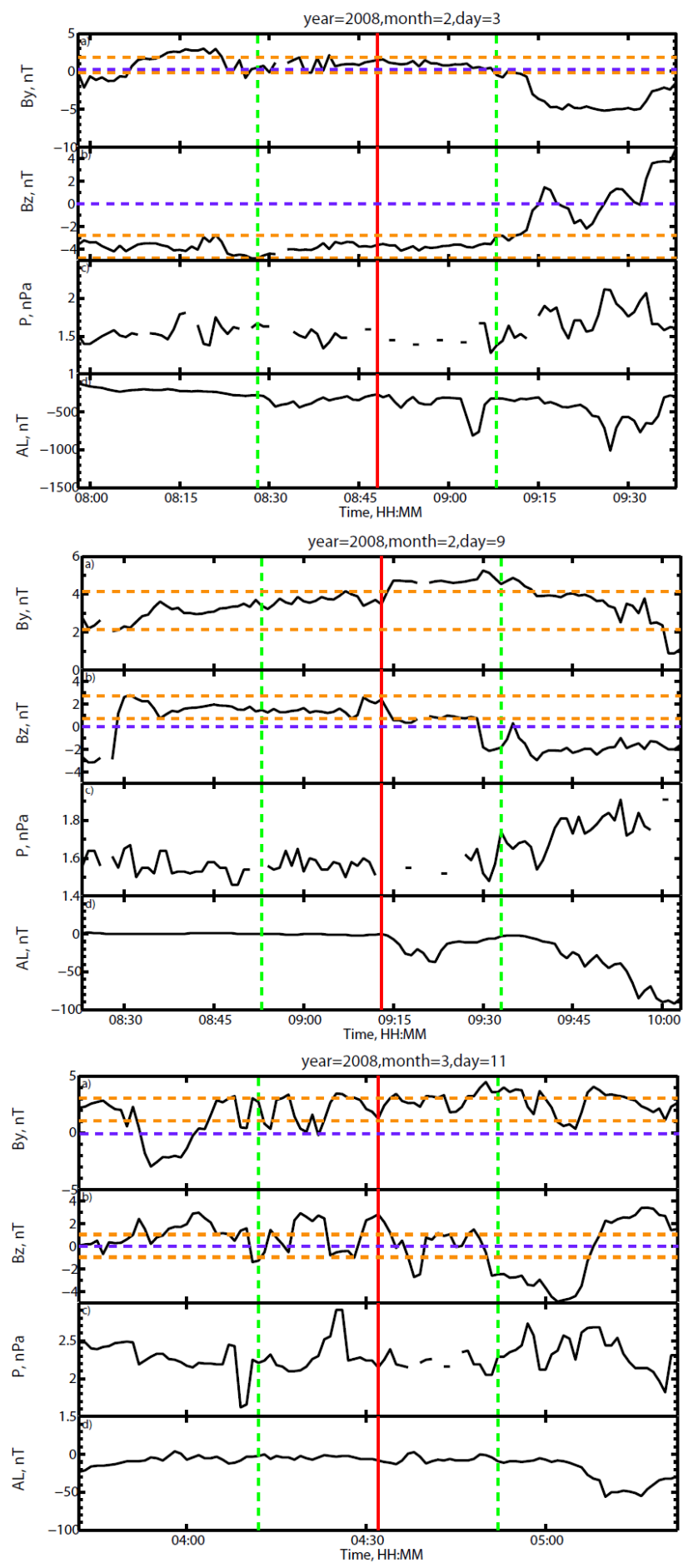

Fig. 3. Examples of Non-triggered events in the same format as Fig. 1.

It is interesting that the triggering percentage is larger for all criteria for the IMAGE-FUV events than for the THEMIS ASI events, despite the effect that substorms are more accurately distinguishable from other auroral activation by the THEMIS ASI. However, the ground-based ASIs are far more

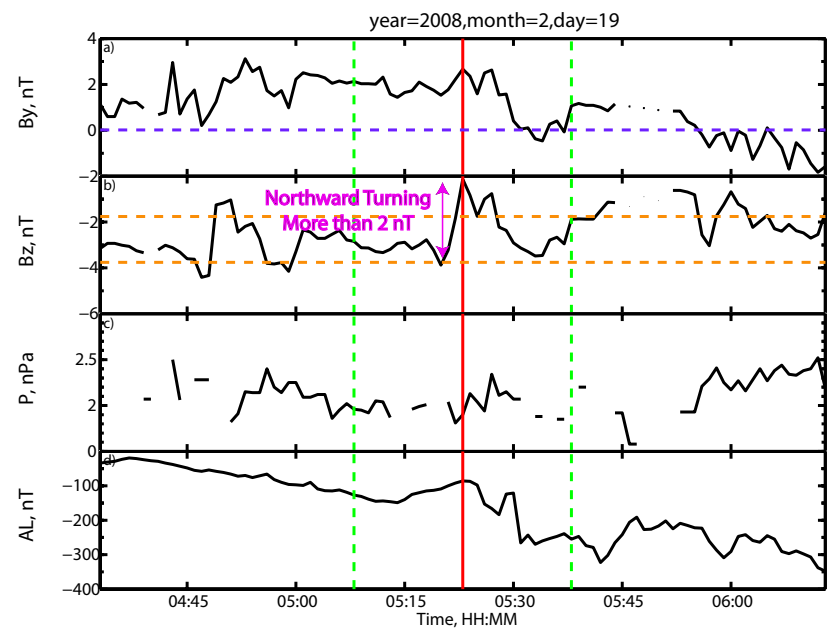

Fig. 4. Example of a $B_{\mathrm{Z}}$-triggered event applying the criteria based on Lyons et al. and the visual criteria in Hsu et al. in the same format as Fig. 1.

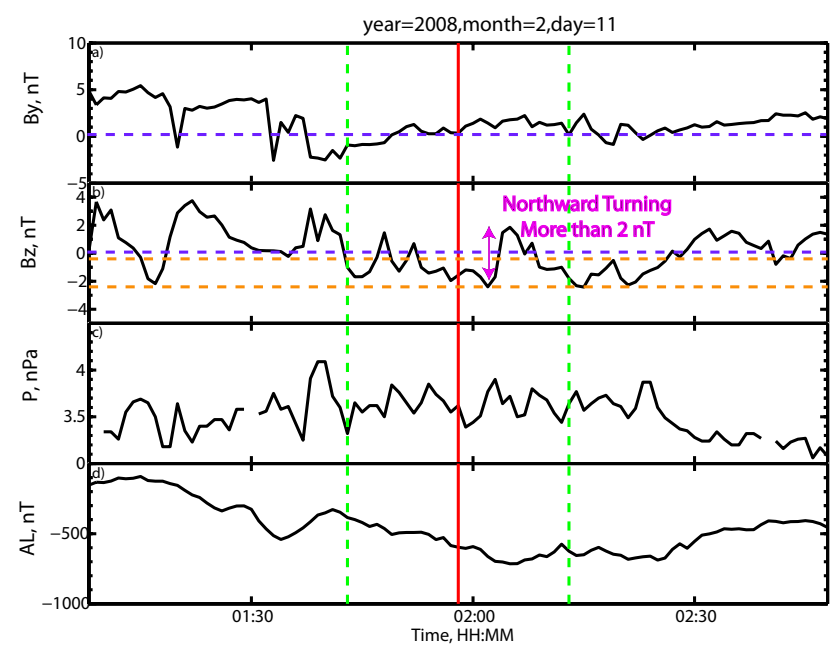

Fig. 5. Example of a $B_{\mathrm{Z}}$-triggered event applying the criteria based on Lyons et al. and the visual criteria in Hsu et al. without requirements for the southward IMF growth phase in the same format as Fig. 1.

sensitive to small substorm auroral activations than in the spacecraft IMAGE-FUV camera, suggesting that it may be easier to identify IMF triggers for larger events or that larger events are more likely to be triggered than are smaller events. To examine this possibility, we first considered the magnitude of the AL index drop associated with the onset However, perhaps due to the sparsity of AL stations, an AL dependence was not observed, and in fact little or no AL response was observed for many onsets. We thus examined the list of large geosynchronous dispersionless injections.

Table 3 clearly shows that the percentage of the IMF triggering increases substantially as compared to Tables 1 and 2 , 
the highest percentage reaching up to $63 \%$. This result leads us to suggest that it may indeed be possible that IMF triggering may be easier to identify for larger events or that larger events are more likely to be triggered than are smaller events. It also interesting that the percentage of non-triggering substorms that occur under mostly southward IMF conditions increases with substorm size, which is consistent with the idea that larger substorms are more likely to be non-triggered under southward IMF conditions than for the other IMF conditions.

\section{Conclusions}

We have evaluated the external substorm triggering by IMF orientation changes, using three different criteria and three substorm onset lists. We have found that the triggering percentage is limited to $17 \%$ and $22 \%$ when applying the Lyons et al. criteria for the THEMIS ASI and IMAGE-FUV event lists, respectively. The percentage increases slightly by including IMF $B_{\mathrm{y}}$ triggering. More interestingly, the relaxed triggering criteria which follow the Hsu et al. method but without requirements for southward IMF prior onset, give significantly higher percentages, reaching close to $50 \%$ (41 and $48 \%$ for the THEMIS and IMAGE lists including the $B_{\mathrm{y}}$ triggering). Our analysis does not allow us to ascertain whether or not the relaxation of the criteria indicates that the real triggering percentage is larger than given by the Lyons et al. criteria. However, our results suggest that this is a possibility that should be considered, particularly because the Lyons et al. criteria were based on very few events.

We have also investigated substorm external triggering for large, dispersionless injections at the geosynchronous orbit, in order to examine if it is possible that the IMF variation leading to substorm can be more pronounced for large substorms. The triggering percentage is found to reach up to $63 \%$ for the relaxed criteria, and $34 \%$ even using the Lyons et al. criteria. This remarkable increase suggests that the external triggering can be more easily found for larger substorms than for smaller substorms, a possibility that warrants further considerations. This tendency leads us to suggest that it is possible that the triggering condition for some events in the THEMIS and IMAGE lists may be missed due to short duration changes and the artificial triggering conditions we have used. Our results suggest that more work is warranted on both the determination of realistic triggering criteria and on the possibility that triggers are more easily identified or triggering is more common for larger than for smaller substorm events. We have also found evidence that larger substorms may be more likely to be non-triggered under mostly southward IMF conditions than for other IMF conditions.

Acknowledgements. We thank T.-S. Hsu and R. L. McPherron for very helpful discussions. This work at UCLA was supported by a Chilean Ministry of Education Scholarship (Becas Chile), National Science Foundation grant ATM-0646233, and NASA grant
NNX09AI06G. Solar wind data and energetic particle data were obtained courtesy of the SPDF OMNIWeb database and the LANL Energetic Particle Team, respectively.

Topical Editor R. Nakamura thanks A. De Jong and another anonymous referee for their help in evaluating this paper.

\section{References}

Caan, M., McPherron, R., and Russell, C. T.: Substorm and Interplanetary Magnetic Field Effects on the Geomagnetic Tail Lobes, J. Geophys. Res., 80, 191-194, 1975.

Caan, M. N., McPherron, R. L., and Russell, C. T.: Characteristics of the association between interplanetary magnetic field and substorms, J. Geophys. Res., 82, 4837-4842, 1977.

Cox, D. R.: Some statistical methods connected with series of events, J. R. Stat. Soc., Ser. B, 17, 129-164, 1955.

Foster, J., Fairfield, D., Ogilvie, K., and Rosenberg, T.: Relationship of Interplanetary Parameters and Occurrence of Magnetospheric Substorms, J. Geophys. Res., 76, 6971-6975, 1971.

Frey, H. U., Mende, S. B., Angelopoulos, V., and Donovan, E. F.: Substorm onset observations by IMAGE-FUV, J. Geophys. Res., 109, A10304, doi:10.1029/2004JA010607, 2004.

Henderson, M., Reeves, G., Belian, R., and Murphree, J.: Observations of magnetospheric substorms occurring with no apparent solar wind/IMF trigger, J. Geophys. Res., 101, 10773-10791, 1996.

Hsu, T.-S. and McPherron, R. L.: An Evaluation of the Statistical Significance of the Association between Northward Turnings of the IMF and Substorm Expansion Onsets, J. Geophys. Res., 107, 1398, doi:1310.1029/2000JA000125, 2002.

Hsu, T.-S. and McPherron, R. L.: Occurrence frequencies of IMF triggered and nontriggered substorms, J. Geophys. Res., 108, 1307, doi:10.1029/2002JA009442, 2003.

Kim, H.-J., Lyons, L. R., Zou, S., Boudouridis, A., Lee, D.Y., Heinselman, C., and McCready, M.: Evidence that solar wind fluctuations substantially affect the strength of dayside ionospheric convection, J. Geophys. Res., 114, A11305, doi:10.1029/2009JA014280, 2009.

Lyons, R. L., Blanchard, G. T., Samson, J. C., Lepping, R. P., Yamamoto, T., Moretto, T.: Coordinated observations demonstrating external substorm triggering, J. Geophys. Res., 102, 2703927051, 1997.

Lyons L. R., Kim, H.-J., Xing, X., Zou, S., Lee, D.-Y., Heinselman, C., Nicolls, M. J., Angelopoulos, V., Larson, D., McFadden, J., Runov, A., and Fornacon, K.-H.: Evidence that solar wind fluctuations substantially affect global convection and substorm occurrence, J. Geophys. Res., 114, A11306, doi:10.1029/2009JA014281, 2009.

McPherron, R. L., Terasawa, T., and Nishida, A.: Solar wind triggering of substorm expansion onset, J. Geomagn. Geoelectr., 38, 1089-1108, 1986.

Morley, S. K. and Freeman, M. P.: On the association between northward turnings of the interplanetary magnetic field and substorm onsets, Geophys. Res. Lett., 34, 08104, doi:08110.01029/02006GL028891, 2007.

Newell, P. T. and Liou, K.: Solar Wind Driving and Substorm Triggering, J. Geophys. Res., 116, A03229, doi:10.1029/2010JA016139, 2011. 
Nishimura, Y., Lyons, R. L., Zou, S., and Angelopoulos, V.: Substorm triggering by new plasma intrusion: THEMIS allsky imager observations, J. Geophys. Res., 115, A07222, doi:10.1029/2009JA015166, 2010.

Reeves, G. D.: Radial propagation of substorm injections, International Conference on Substorms-3, Eur. Space Agency Spec. Publ., ESA SP-339, 579-584, 1996.

Rostoker, G.: Triggering of Expansive Phase Intensifications of Magnetospheric Substorms by Northward Turnings of the Interplanetary Magnetic Field, J. Geophys. Res., 88, 6981-6993, 1983.
Rostoker, G., Akasofu, S.-I., Foster, J., Greenwald, R. A., Lui, A. T. Y., Kamide, Y., Kawasaki, K., McPherron, R. L., and Russell, C. T.: Magnetospheric substorms - Definition and signatures, J. Geophys. Res., 85, 1663-1668, doi:10.1029/JA085iA04p01663, 1980.

Samson, J. C., Lyons, L. R., Newell, P. T., Creutzberg, F., and Xu, B.: Proton aurora and substorm intensifications, Geophys. Res. Lett., 19, 2167-2170, doi:10.1029/92GL02184, 1992. 